\title{
Rabbit Ear Membranes as an Interesting Alternative for Permeability Tests in the Preformulation Stages of Cosmetic Products
}

\author{
Estefania Benavides ${ }^{1}$, Sara Manjarres ${ }^{1}$, Constain H. Salamanca ${ }^{1, *(1)}$ and \\ Alvaro Barrera-Ocampo ${ }^{2, *}$ \\ 1 Laboratorio de Diseño y Formulación de Productos Químicos y Derivados, Facultad de Ciencias Naturales, \\ Universidad ICESI, Calle 18 No. 122-135, Cali 760035, Colombia; estefania.benavidesg@hotmail.com (E.B.); \\ sara.manjarres@hotmail.com (S.M.) \\ 2 Departamento de Ciencias Farmacéuticas, Facultad de Ciencias Naturales, Universidad ICESI, \\ Calle 18 No. 122-135, Cali 760035, Colombia \\ * Correspondence: chsalamanca@icesi.edu.co (C.H.S.); aabarrera@icesi.edu.co (A.B.-O.)
}

Received: 6 April 2020; Accepted: 12 May 2020; Published: 18 May 2020

\begin{abstract}
In the pre-formulation stages of cosmetic products, in vitro permeability tests correspond to an important alternative for making better decisions regarding the stability, performance and biocompatibility of these products. Moreover, these tests allow us to establish whether a cosmetic ingredient can penetrate the different layers of the skin, which is essential in these products. This study was focused on the extraction, characterization and preservation of rabbit ear membranes and their subsequent performance comparison against two synthetic membranes (cellulose and Strat-M ${ }^{\mathrm{TM}}$ ). For this, the rabbit ear stratum corneum was isolated and characterized histologically, using the Hematoxylin and Eosin (HE) staining protocol along with light microscopy and image analysis. Then, the Frank diffusion cell (FC) model was employed to evaluate and compare the permeability of the model compound Naproxen sodium (NPX) between the three membrane systems. The results show that NPX permeability is strongly affected by the type of membrane, and the implementation of rabbit ear membranes shows high reproducibility, demonstrating that this model could be implemented during pre-formulation studies of cosmetic products.
\end{abstract}

Keywords: cosmetic pre-formulation stages; Franz diffusion cell; permeability assay; rabbit ear membranes; Naproxen sodium

\section{Introduction}

For the year 2018, it was estimated that the growth of the cosmetic market was close to 5.5\%, thus becoming one of the fastest growing industries in the world. Skin care products are the most profitable category in the sector and, by 2019, represented more than $39 \%$ of the global cosmetic market with a projected growth of around USD 20.1 billion [1]. For many skin care products, permeability is a fundamental property necessary for the product to carry out the effect promised to customers in the claims. Furthermore, the permeability studies allow for verifying the degree to which a cosmetic ingredient can penetrate the different skin layers and therefore guarantee the proper performance of this kind of product. The permeability reflects the capacity or speed with which a molecule can cross a membrane, depending on its characteristics, as well as the physicochemical properties of the vehicle (cosmetic formulation), specifically lipophilicity, load and size [2]. From this, the cosmetic permeability studies have allowed to obtain information about the absorption mechanisms in pre-formulation stages, since the interaction between the cosmetic product depends on the skin, the penetration into the stratum corneum and penetration into skin layers [3,4]. To evaluate the permeability of compounds of 
interest in cosmetic formulations, techniques based on in vitro models have been developed, which can be considered a great advance in the cosmetic industry, since the use of biomodels for the verification of claims has decreased considerably. However, some of the caveats of these models are the low reproducibility and the lack of similarity with the human skin. As a way to overcome these drawbacks, in vitro models have evolved thanks to the development of synthetic membranes, cell lines and the use of preserved membranes obtained as a by-product from animals destined for human consumption or for other purposes [5].

Franz cells (FC) are an in vitro methodology that allows for evaluating the permeability by the transdermal diffusion of topical formulations such as gels, creams, ointments and patches [6-8]. In this model, the passage of a substance from a donor compartment (upper part of the cell) to an acceptor compartment (lower part of the cell) is evaluated. In the middle of both compartments is a membrane that can be of synthetic or biological origin. Synthetic membranes are widely used in permeability studies because they are commercially available. However, most of these can only emulate the hydrophilic or hydrophobic characteristics of human skin, but not both at the same time [9]. An example of this is cellulose acetate membranes [10], which act as a porous barrier without limiting the rate of permeability. Additionally, there is a risk that they interact with the components of the formulation altering the results of the tests. Strat- $\mathrm{M}^{\mathrm{TM}}$ membranes arise as a response to this dichotomy; these are polymeric membranes made of highly packed multi-layered polyether sulfone, thus allowing for the evaluation of the permeability of both hydrophilic and hydrophobic compounds, closely resembling the behavior of human skin [11,12]. On the other hand, membranes of biological origin represent a valuable alternative due to their proximity in the structural composition and the biophysical properties with those of human skin. Membranes from pigs, rabbits, rats and mice have been used to perform permeability tests, and, thanks to the development of tissue preservation techniques, it is now possible to store these membranes for long periods of time without the need for specialized equipment [13-17]. Therefore, in this study, it was developed an in vitro methodology to evaluate the permeability of ingredients of interest for cosmetic formulations. Besides, FC was used to study the permeability of the model molecule naproxen (NPX) through three types of membranes, two of synthetic origin (cellulose and Strat- $\mathrm{M}^{\mathrm{TM}}$ ) and one of biological origin (rabbit ear). For this, the extraction and conservation of stratum corneum membranes (SCM) of the rabbit ear were standardized. The performance of SCM was compared with that of synthetic membranes. The results show that NPX permeability through SCM and Strat-M ${ }^{\mathrm{TM}}$ membranes is comparable, but it is different from cellulose membranes, thus emphasizing the importance of both biological and polymeric multi-layered membranes in permeability assays during the pre-formulation studies of cosmetic products.

\section{Methodology}

\subsection{Chemicals}

The reagents and materials used in this study were: Naproxen sodium given by Tecnoquimicas laboratory (Cali-Colombia), $\mathrm{NaOH}, \mathrm{KH}_{2} \mathrm{PO}_{4}, \mathrm{~K}_{2} \mathrm{HPO}_{4}, \mathrm{NaH}_{2} \mathrm{PO}_{4}, \mathrm{Na}_{2} \mathrm{HPO}_{4}, 4 \%$ formaldehyde, n-hexane, (50\%-100\%) ethanol, xylene, paraffin (Paraplast X-tra) and isopropanol 1\% were obtained from Sigma-Aldrich (St. Louis, MO, USA), 10X trypsin (Gibco, Waltham, MA, USA), Harris hematoxylin and eosin from BiopharChem (Hatfield, AK, USA). Ultra-pure water (pH 5.5 and conductivity $1 \mu \mathrm{S} / \mathrm{cm}$ ) was obtained from the Ellix Essencial Millipore Purification System ${ }^{\mathrm{TM}}$ (Darmstadt, Germany).

\subsection{Membranes}

Cellulose acetate dialysis membranes were purchased from Sigma-Aldrich (St. Louis, MO, USA), Strat-M $\mathrm{M}^{\mathrm{TM}}$ membranes from Millipore (Temecula, MA, USA). Ear membranes from 4 male New Zealand breed rabbits weighing between 2.30 and $3.80 \mathrm{~kg}$ (average weight $3.26 \mathrm{~kg}$ ) were obtained from the Tissue Bank of the Animal Facility of Universidad Icesi (Cali, Colombia). The ears were dissected 
from corpses of rabbits used in other research projects. This was conducted with the approval of the Institutional Animal Ethical Committee of the Universidad Icesi (Cali, Colombia). In order to isolate de stratum corneum of the rabbit's ear, the inner part of the ear was separated by the cartilage from the skin; the sectioned area was about $6 \mathrm{~cm} \times 4 \mathrm{~cm}$ approximately. A section of $5 \mathrm{~mm} \times 5 \mathrm{~mm}$ of each ear membrane was placed in $4 \%$ formaldehyde for $24 \mathrm{~h}$ and used for histology experiments (Figure 1). The rest of the ear was deposited in sodium phosphate buffer at $\mathrm{pH} 7.4$ for subsequent preservation treatment.

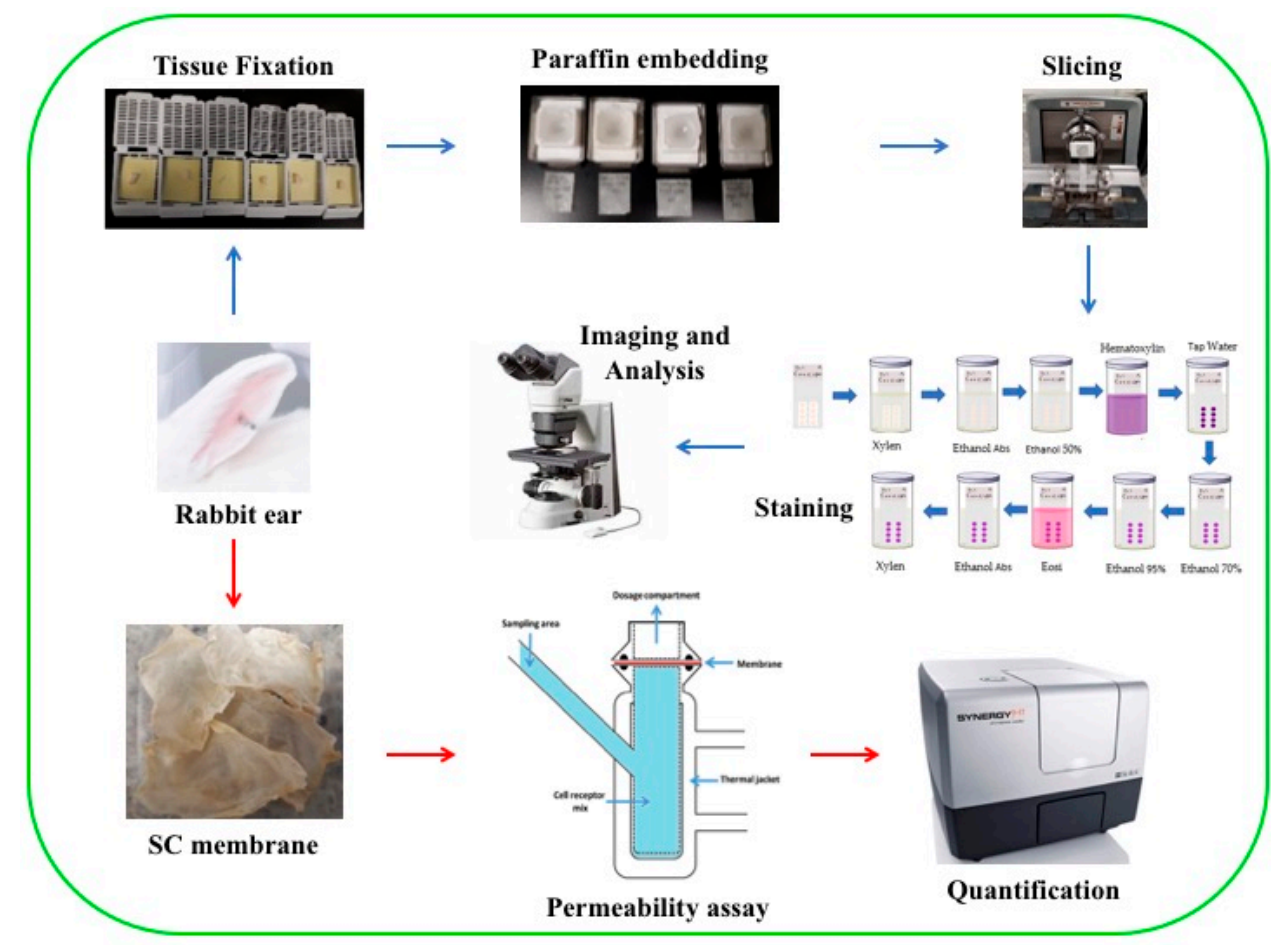

Figure 1. Schematic of tissue characterization and permeability assays using stratum corneum membranes (SCM) obtained from rabbit ears and compared with synthetic membranes.

\subsection{Conservation of Rabbit'S Ear Stratum Corneum}

The SCM were introduced in hot water at $60^{\circ} \mathrm{C}$ for two minutes to separate the epidermis from the dermis. The epidermal sheets were moistened in a 1\% trypsin solution and sodium phosphate buffer $\mathrm{pH} 7.4$ for $15 \mathrm{~h}$ at $4{ }^{\circ} \mathrm{C}$. After this time, the stratum corneum sheets were washed individually with distilled water. The tissue was incubated in $1 \%$ trypsin solution for two hours at $37^{\circ} \mathrm{C}$ and was washed a second time with distilled water. Then, the samples were immersed in n-hexane for 5 min and washed with distilled water. Finally, the pieces of tissue were put in the drying oven for two hours at $37^{\circ} \mathrm{C}$ and stored in a desiccator until use [18].

\subsection{Histology}

Sections of SCM were placed in histology cassettes and dehydrated by a series of ethanol solutions of increasing concentration (50\%,70\%, 90\% and 95\%) during $1 \mathrm{~h}$ each. After this, the tissue was incubated in isopropanol, then xylene and finally in paraffin for $2 \mathrm{~h}$ in each solution. Paraffin was melted at $58{ }^{\circ} \mathrm{C}$ and it was used for the embedding of the SCM. The tissue was cut using a microtome to obtain $5 \mu \mathrm{m}$ sections, which were extended for $5 \mathrm{~min}$ in a hot bath $\left(42{ }^{\circ} \mathrm{C}\right)$ with $1 \%$ gelatin, then the samples were mounted on slices and dried in an oven at $45^{\circ} \mathrm{C}$ for $2 \mathrm{~h}$ (Figure 1 ). SCM sections were deparaffined and rehydrated using xylene, absolute ethanol and 50\% ethanol for $3 \mathrm{~min}$. The tissue was stained with Harris hematoxylin during $4 \mathrm{~min}$, and then it was washed with distilled water and placed in $70 \%$ and $95 \%$ ethanol for $20 \mathrm{~s}$. After that, the tissue was transferred to $0.5 \%$ esosin solution, and next 
placed in absolute ethanol and xylene for $20 \mathrm{~s}$. Cover slips were applied using mounting medium (Thermo Scientific Shandon Consul-Mount) (Figure 1).

\subsection{Light Microscopy and Image Analysis}

SCM sections were observed with a $4 \mathrm{X}$ magnification objective in a Nikon Eclipse 55i microscope (Melville, NY, USA) and images were acquired using a Nikon Digital Sight D5-U3 camera (Melville, NY, USA). The area of the SCM was measured using the free distribution software Fiji [19].

\subsection{Preparation of NPX formulation}

In this case, an extremely simple formulation was developed with the objective that the drug permeability would depend exclusively on the type of membrane and not on other extrinsic factors of the formulation. Consequently, a $\%$ NPX hydroalcoholic solution was made. For this, $1 \mathrm{~g}$ of NPX was weighed and added to $20.8 \mathrm{~mL}$ of $96 \%$ ethanol and $29.2 \mathrm{~mL}$ of phosphate buffer (pH 5.5 and $0.15 \mathrm{M}$ ionic strength). Subsequently, the blend was stirred at $25^{\circ} \mathrm{C}$ and $300 \mathrm{rpm}$ until the formation of the NPX solution. Likewise, it is important to mention that Naproxen sodium was chosen as a model molecule, because it has been previously used for other permeability studies [20-22] and mainly because it is a very stable molecule in aqueous medium, which is fundamental for this type of assay.

\subsection{Methods}

\subsubsection{Determination of NPX Permeability}

The experiments were carried out in one FC with a nominal volume in the acceptor compartment of $120 \pm 2 \mathrm{~mL}$ and a diffusion area of $5.7 \mathrm{~cm}^{2}$. A total of $5 \mathrm{~mL}$ of the $2 \%$ NPX solution was placed in the donor compartment. The experiments were carried out in triplicate at a temperature of $37^{\circ} \mathrm{C}$ and $480 \mathrm{rpm}$ for 8 hours. Firstly, a cellulose acetate membrane of $2.7 \mathrm{~cm}$ in diameter, $76 \mathrm{~mm}$ in size and a pore of $45 \mu \mathrm{m}$ was used, which was hydrated for 24 hours in $\mathrm{pH} 5.5$ phosphate buffer and $0.15 \mathrm{M}$ ionic strength. The acceptor compartment was completed with phosphate buffer at a temperature of $37^{\circ} \mathrm{C}$ previously degassed for two hours and stirring was adjusted. The samples were evaluated at different points and the data analysis was done according to the permeability efficiency (PE). The tests were conducted under physiological conditions corresponding to the phosphate buffer pH 5.5 at $0.15 \mathrm{M}$. In addition, three types of membranes were used: a cellulose acetate dialysis membrane with a molecular weight cut-off of $12 \mathrm{kDa}$, a Strat- $\mathrm{M}^{\mathrm{TM}}$ membrane and the SCM membrane from rabbit ear.

On the other hand, the comparison of NPX permeability with respect to the three types of membranes was analyzed using the permeability efficiency (PE) parameter [6]. Such a parameter is obtained from the area under the curve (AUC) of the permeated molecule concentration vs. time profile in the Franz diffusion cell. Therefore, such a parameter allows one to establish a gradient of ability of the molecule to permeate establishing values between $0 \%$ (non-permeation) and $100 \%$ (complete permeability). Such parameter can also be used to establish the behavior of different types of membranes against a specific molecule, as happened in this study. So, the PE parameter was obtained according to:

$$
\mathrm{PE}=\frac{\mathrm{AUC}}{\mathrm{R}} \times 100 \%=\frac{\int_{0}^{\mathrm{t}} \mathrm{y}_{\mathrm{x}} \mathrm{dt}}{y_{100} \mathrm{xt}} \times 100 \%
$$

where $y_{100}$ is the value of the AUC assumed with a permeation of $100 \%$ in a time interval, $t$, and $y$ represents the AUC value of the permeated drug during the same time interval.

\subsubsection{NPX Quantification}

The quantification of NPX was performed using the Synergy H1 plate reader at $272 \mathrm{~nm}$. From the acceptor compartment, $500 \mu \mathrm{L}$ was taken in a defined time interval. To guarantee the sink conditions of the experiment, the amount extracted was replenished with $\mathrm{pH} 5.5$ phosphate buffer in order to 
keep the initial volume constant. At the end of the experiment, $150 \mu \mathrm{L}$ of each sample was taken and served in a 96-well plate. From this measure, it was evaluated that the coefficient of variation should not exceed $5 \%$ in order to guarantee the reproducibility of the method.

\subsubsection{Statistical Analysis}

All the data were tabulated in Microsoft Excel ${ }^{\text {TM }} 2016$ (Redmond, WA, USA), and the statistical analysis was performed using the software GraphPad Prism 8.0 (San Diego, CA, USA). A non-parametric Friedman test was applied in order to compare whether there were significant differences between the area of the serial cuts of the SCM. This same test was used to analyse if there were differences between the SCM thickness of the 4 rabbits. To determine the differences between independent groups, Dunn's multiple comparisons post-hoc test was applied where a 95\% confidence level was adopted, and the data were presented as mean \pm standard deviation.

The data obtained during the permeability assays were analysed for homogeneity of variances using the Bartlett test and the changes of permeability efficiency (PE) values in time were compared using a two-way ANOVA. To determine the differences between independent groups, the Tukey post-hoc test was applied where a 95\% confidence level was adopted, and the data were presented as mean \pm standard deviation.

\section{Results and Discussion}

Various guides have been published by government agencies and specialized laboratories around the world in order to determine the percutaneous permeability and absorption of chemical compounds [23-25]. Although the methodologies proposed by these guidelines are valid, some are difficult to implement in research and development laboratories for cosmetic products. This is because some of the guidelines recommend the use of radioactively labelled compounds and their purpose is to evaluate the acute and chronic toxicity of chemical substances once they have passed through the stratum corneum, the skin layers and their absorption into the bloodstream [26,27]. In addition, most of the guidelines recommend the use of rat skin in absorption tests. However, rodent skin seems to have a thinner stratum corneum and is more permeable than human skin. By contrast, although the epidermis and dermis of rabbit ear skin are thinner than those of human and pig, the thickness of the stratum corneum is comparable between the three species [5]. Therefore, this study focused on the implementation of a methodology to obtain SCM from rabbit ear and evaluate its use in cosmetic preformulation studies when compared to two membranes of synthetic origin commercially available.

\subsection{Extraction and Conservation of Biological Membranes}

The rabbit ear membranes obtained showed good behaviour when storing and using them. These could be preserved indefinitely without altering their characteristics and the appearance of fungi on its surface was not observed. In addition, by hydrating them they maintained their initial properties, allowing them to be used successfully in FC. Figure 1 shows the result of the conservation process.

\subsection{Histological Characterization of Rabbit Ear SCM}

Four rabbit ears were used in this study. Serial cuts of $5 \mu \mathrm{m}$ were made in each sample. These sections were stained with Hematoxylin and Eosin (HE) and subsequently observed under a light microscope using a $4 \mathrm{X}$ objective. Images of the serial sections were captured using the Fiji software and the area between the cartilage and the outer membrane of each section was measured (Figure 2a). The measured area represents the stratum corneum, which is the most superficial layer of the epidermis of the rabbit ear (Figure 2a). Four sections of each sample were analysed, and the data were subjected to statistical analysis. Friedman's test revealed that there was no statistically significant difference between the areas of the samples (mean area: $216.2 \pm 10.2 \mu \mathrm{m}^{2}, \mathrm{CV}: 4.7 \%$ ) (Figure $2 \mathrm{~b}$ ). These same images were used to determine the thickness of the serial sections of the membranes obtained from the ears of the four rabbits (Figure 2c). Statistical analyses show that there are no statistically significant 
differences in the thickness of SCM used in this study (mean thickness: $14.7 \pm 0.4 \mu \mathrm{m}, \mathrm{CV}: 2.7 \%$ ). These results indicate that the stratum corneum of the rabbit ears used in this study had a comparable area and thickness, which represents an important parameter due to the variability that this type of sample usually has.

a)

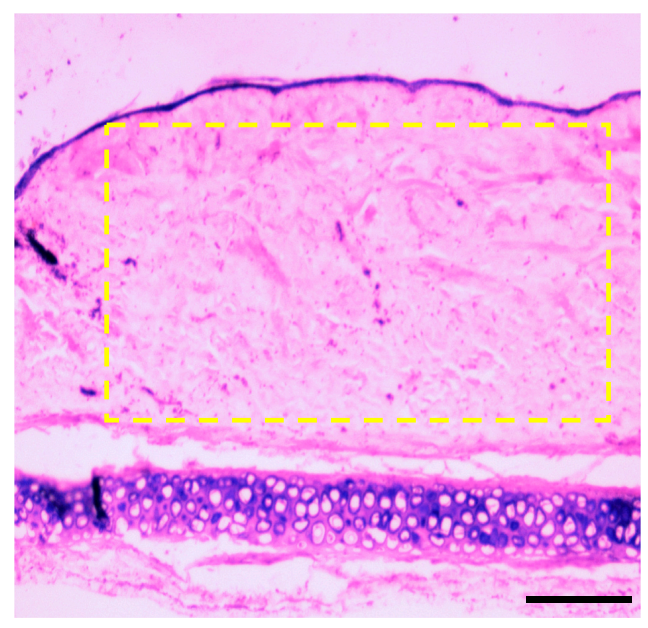

b)

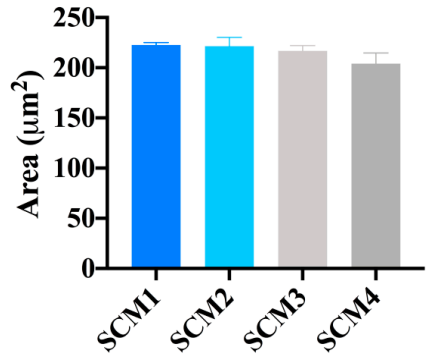

c)

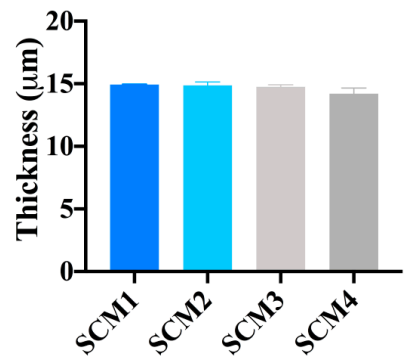

Figure 2. Measurements of rabbit ear membranes, where (a) representative tissue section depicting the area of the stratum corneum that it was quantified, Scale bar: $5 \mu \mathrm{m}$; (b) histogram of the area measurements of serial sections of the SCM obtained from 4 different rabbits; (c) histogram of the thickness measurements of serial sections of the SCM obtained from 4 different rabbits.

The thickness of SCM from rabbit ear obtained in this study is in agreement with the values reported by other authors [18] and it resembles closely to the thickness of human stratum corneum [28]. When compared, SCM are thinner than Strat-M $\mathrm{M}^{\mathrm{TM}}$ membranes $(14.7 \mu \mathrm{m}$ vs. $300 \mu \mathrm{m})$ [12] and have a much more complex composition in terms of the layers of cells and macromolecules. For instance, it has been reported that SCM from rabbit ear obtained with the same protocol used in this study is composed by ceramides ( $\alpha$-hydroxyl), free fatty acids (oleic acid), cholesterol, cholesterol esters (cholesterol oleate) and triglycerides (triolein) [18]. By contrast, Strat- $\mathrm{M}^{\mathrm{TM}}$ membranes mimic the layer of human skin by increasing porosity and thickness. These synthetic membranes are made of polyethersulfone, polyolefin and lipids, which include ceramides, cholesterol and free fatty acids similar to what is found in the human skin [12]. Despite the differences in thickness, the lipid composition of both types of membranes is comparable, giving them characteristics for the transport of hydrophilic and lipophilic molecules. In addition, the intricate arrangement of cell layers and pores appears to slow the transit of nonpolar molecules such as NPX [29,30]. On the other hand, Cellulose membranes are thicker $(80-160 \mu \mathrm{m})$ than SCM and Strat-M $\mathrm{M}^{\mathrm{TM}}$ membranes and the pore size is larger than that of human skin, displaying higher permeation than the multi-layered membranes used in this study [31,32].

\subsection{Quantification of NPX Concentration}

The results show that the methodology adopted to quantify the percentage of NPX released during the permeability experiment has a high level of reproducibility to the linear heuristic model $\left(\mathrm{Y}=8.33 \mathrm{x}+0.0154\right.$ with $\left.\mathrm{R}^{2}=0.999\right)$.

\subsection{Determination of NPX Release Profile}

The in vitro release profiles of NPX for 8 hours were determined using FC and the three different membranes in study. Initially, the experiment was carried out in cellulose acetate membranes, and the 
results show that NPX reaches a maximum release percentage of 18.19 in the first 8 hours of experiment (Figure 3). In contrast, after 8 hours, the crossing of NPX through Strat-M ${ }^{\mathrm{TM}}$ and SCM membranes was very limited, reaching a maximum release percentage of 6.35 and 5.90, respectively (Figure 3).

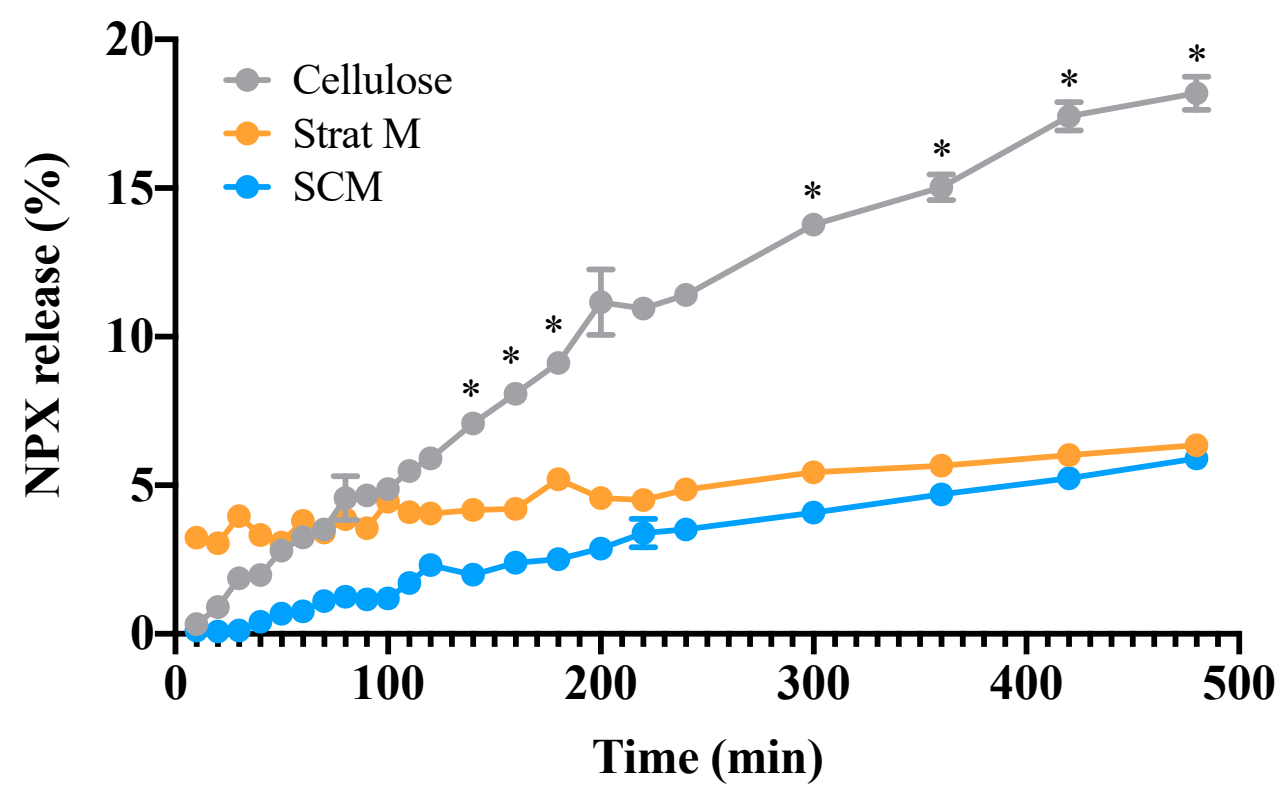

Figure 3. Naproxen sodium (NPX) release profiles in Franz cells (FC). The release of a hydroalcoholic solution of NPX through cellulose, Strat-M $\mathrm{M}^{\mathrm{TM}}$ and SCM membranes was followed for $8 \mathrm{~h}$.

The release profiles show a marked dependence on the type of membrane used in the FC. When using cellulose acetate membranes, the concentration of the NPX is a function of time, and only after 8 hours does the release seem to reach a plateau (Figure 3). This contrasts the release profile of NXP observed with Stat-M ${ }^{\mathrm{TM}}$ and SCM membranes. The results show that the crossing of NPX through both types of membranes is a slow process, thus reaching only $30 \%$ of the percentage released by cellulose membranes. Data analysis reveals a statistically significant difference between the release percentage of NPX from cellulose acetate and the other two types of membranes just after 140 min of experiment $(p<0.001)$, and differences remain until the end of the experiment.

\subsection{Evaluation of NPX Permeability}

The permeability efficiency was determined by means of the area under the curve (AUC) where the assumption is of a concentration of $100 \%$ (Table 1 ). Figure 3 shows that the release of NPX is very low, considering that the hydroalcoholic solution had a concentration of $2 \%(\mathrm{w} / \mathrm{v})$. This behavior may be explained by the non-polar nature of NPX, which is more related to the solution present in the donor compartment than to the phosphate buffer $\mathrm{pH} 5.5$ with $0.15 \mathrm{M}$ ionic strength disposed in the acceptor compartment. In addition, it is a molecule with a low solubility in aqueous solutions. The experiments were performed using phosphate buffer $\mathrm{pH} 5.5$ in order to mimic the $\mathrm{pH}$ of the human skin. At this $\mathrm{pH}$, the ionization state of NPX is mostly neutral (the pKa value for NPX is 4.15) and penetrates more easily the structurally simpler cellulose acetate membranes, while this transit is more delayed in membranes with complex layers, such as Strat- $\mathrm{M}^{\mathrm{TM}}$ and SCM; thus, for the model compound to permeate throughout, it needs to overcome different layers, which takes more time in the FC experiment. 
Table 1. Permeability efficiency of NPX using FC with cellulose, Strat-M ${ }^{\mathrm{TM}}$ and SCM membranes.

\begin{tabular}{cc}
\hline Membrane & Permeability Efficiency (\% PE) \\
\hline Cellulose & 10.92 \\
Strat-M & 4.91 \\
SCM & 3.28 \\
\hline
\end{tabular}

The PE of NPX (Table 2) in the FC using Strat-M $\mathrm{M}^{\mathrm{TM}}$ membrane is comparable with that of the biological membranes (3.28\% vs. $4.91 \%$ ) (Table 2). Strat-M $\mathrm{M}^{\mathrm{TM}}$ membranes are very close to human skin, because it is composed of polymeric layers that create a porous structure impregnated with a proprietary blend of synthetic lipids, which confers skin-like properties (non-polar conditions) additional to the synthetic membrane [30], that is, it contains a combination of lipids in a specific proportion similar to that found in the human stratum corneum $[33,34]$. Allowing for a simulation closer to the physiological conditions of humans, giving more accurate results, and a simulation closer to the in vivo model. However, PE values of these two membranes are significantly low compared to that of cellulose acetate membranes ( $3.28 \%$ and $4.91 \%$ vs. $10.92 \%$ ) (Table 1 ).

Table 2. Comparison of permeability efficiencies between cellulose acetate, Strat-M ${ }^{\mathrm{TM}}$ and SCM membranes using the FC in vitro model.

\begin{tabular}{cc}
\hline Tukey Multiple Comparison Test & Adjusted P Value \\
\hline Cellulose vs. Strat $\mathrm{M}^{\mathrm{TM}}$ & 0.0039 \\
Cellulose vs. SCM $^{\mathrm{TM}}$ vs. SCM & 0.0041 \\
Strat $^{\mathrm{T}}$ & 0.9985 \\
\hline
\end{tabular}

\section{Conclusions}

In this study, a methodology for the extraction and long-term conservation of biological membranes was implemented, which showed comparable characteristics and performance with synthetic membranes during the experiments with FC. In this model, the selection of the membrane is a critical step because generic membranes such as regenerated cellulose can provide evidence on important changes associated with the size of the molecules evaluated and the physicochemical characteristics of the vehicle used to dissolve it. Human skin-like membranes such a Strat- $\mathrm{M}^{\mathrm{TM}}$ and SCM from rabbit ears provide important information about the permeation changes of cosmetic ingredients used in the pre-formulation phase of product development. Regarding the permeation process of NPX, it was established that this parameter decreases when complex membranes are used, thus reflecting some of the phenomena observed during the permeation process in human skin. Finally, it was demonstrated that the FC model using cellulose, Strat-M ${ }^{\mathrm{TM}}$ and SCM membranes as a characterisation tool can be easily implemented for the pre-formulation stage or during the evaluation of the performance of skin care products and where the SCM membrane describes a behaviour closer to reality.

Author Contributions: C.H.S. designed the experiment and reviewed the first draft of the manuscript and reviewed the final document. A.B.-O. contributed to the execution of the extraction and conservation of rabbit ear membranes, wrote the first draft and the final version of the manuscript. E.B. developed the Naproxen sodium permeability evaluation experiment (NPX), analysed the data and wrote the first draft of the manuscript and S.M. developed the Naproxen sodium permeability evaluation experiment (NPX) and analysed the data. All authors have read and agreed to the published version of the manuscript.

Funding: This research received no external funding.

Acknowledgments: The authors thank the Icesi University for facilitating the spaces in which the research was carried out.

Conflicts of Interest: The authors declare no conflict of interest. 


\section{References}

1. Annual Growth of the Global Cosmetics Market from 2004 to 2019. Available online: https://www.statista. com/statistics/297070/growth-rate-of-the-global-cosmetics-market/ (accessed on 13 May 2020).

2. Avdeef, A. Absorption and Drug Development: Solubility, Permeability, and Charge State, 2nd ed.; Wiley Publisher: New York, NY, USA, 2012.

3. Zsikó, S.; Csányi, E.; Kovács, A.; Budai-Szúcs, M.; Gácsi, A.; Berkó, S. Methods to evaluate skin penetration In Vitro. Sci. Pharm. 2019, 87, 19. [CrossRef]

4. Torin Huzil, J.; Sivaloganathan, S.; Kohandel, M.; Foldvari, M. Drug delivery through the skin: Molecular simulations of barrier lipids to design more effective noninvasive dermal and transdermal delivery systems for small molecules, biologics, and cosmetics. Wiley Interdiscip. Rev. Nanomed. Nanobiotechnol. 2011, 3, 449-462. [CrossRef] [PubMed]

5. Ghosh, T.K.; Walters, K.A.; Lane, M.E. Dermal and Transdermal Drug Delivery Systems. In Dermal Drug Delivery; Taylor \& Francis Inc.: Boca Raton, FL, USA, 2019; pp. 1-447.

6. Salamanca, C.H.; Barrera-Ocampo, A.; Lasso, J.C.; Camacho, N.; Yarce, C.J. Franz diffusion cell approach for pre-formulation characterisation of ketoprofen semi-solid dosage forms. Pharmaceutics 2018, 10, 148. [CrossRef]

7. Godin, B.; Touitou, E. Transdermal skin delivery: Predictions for humans from In Vivo, Ex Vivo and animal models. Adv. Drug Deliv. Rev. 2007, 59, 1152-1161. [CrossRef] [PubMed]

8. Venter, J.P.; Muller, D.G.; Du Plessis, J.; Goosen, C. A comparative study of an in situ adapted diffusion cell and an in vitro Franz diffusion cell method for transdermal absorption of doxylamine. Eur. J. Pharm. Sci. 2001, 13, 169-177. [CrossRef]

9. Barry, B.W. Modern methods of promoting drug absorption through the skin. Mol. Aspects Med. 1991, 12, 195-241. [CrossRef]

10. Barry, B.W.; Brace, A.R. Permeation of oestrone, oestradiol, oestriol and dexamethasone across cellulose acetate membrane. J. Pharm. Pharmacol. 1977, 29, 397-400. [CrossRef]

11. Neupane, R.; Boddu, S.H.S.; Renukuntla, J.; Babu, R.J.; Tiwari, A.K. Alternatives to biological skin in permeation studies: Current trends and possibilities. Pharmaceutics 2020, 12, 152. [CrossRef]

12. Haq, A.; Goodyear, B.; Ameen, D.; Joshi, V.; Michniak-Kohn, B. Strat-M ${ }^{\circledR}$ synthetic membrane: Permeability comparison to human cadaver skin. Int. J. Pharm. 2018, 547, 432-437. [CrossRef]

13. Dezani, A.B.; Pereira, T.M.; Caffaro, A.M.; Reis, J.M.; Serra, C.H. dos R. Determination of lamivudine and zidovudine permeability using a different Ex Vivo method in Franz cells. J. Pharmacol. Toxicol. Methods 2013, 67, 194-202. [CrossRef]

14. Davies, D.J.; Heylings, J.R.; McCarthy, T.J.; Correa, C.M. Development of an in vitro model for studying the penetration of chemicals through compromised skin. Toxicol. Vitr. 2015, 29, 176-181. [CrossRef] [PubMed]

15. Cilurzo, F.; Minghetti, P.; Sinico, C. Newborn pig skin as model membrane in in vitro drug permeation studies: A technical note. AAPS PharmSciTech 2007, 8, 97-100. [CrossRef] [PubMed]

16. Shah, S.N.H.; Shahzad, Y.; Akash, M.S.H.; Ali, M.; Bukhari, S.N.I.; Hassan, S.S.U. Rabbit skin and polydimethylsiloxane as model membranes to evaluate permeation kinetics from topical formulation. Pak. J. Zool. 2013, 45, 159-166.

17. Simon, A.; Amaro, M.I.; Healy, A.M.; Cabral, L.M.; de Sousa, V.P. Comparative evaluation of rivastigmine permeation from a transdermal system in the Franz cell using synthetic membranes and pig ear skin with in vivo-in vitro correlation. Int. J. Pharm. 2016, 512, 234-241. [CrossRef]

18. Nicoli, S.; Padula, C.; Aversa, V.; Vietti, B.; Wertz, P.W.; Millet, A.; Falson, F.; Govoni, P.; Santi, P. Characterization of rabbit ear skin as a skin model for In Vitro transdermal permeation experiments: Histology, lipid composition and permeability. Skin Pharmacol. Physiol. 2008, 21, 218-226. [CrossRef]

19. Schindelin, J.; Arganda-Carreras, I.; Frise, E.; Kaynig, V.; Longair, M.; Pietzsch, T.; Preibisch, S.; Rueden, C.; Saalfeld, S.; Schmid, B.; et al. Fiji: An open-source platform for biological-image analysis. Nat. Methods 2012, 9, 676-682. [CrossRef]

20. Zakeri-Milani, P.; Barzegar-Jalali, M.; Tajerzadeh, H.; Azarmi, Y.; Valizadeh, H. Simultaneous determination of naproxen, ketoprofen and phenol red in samples from rat intestinal permeability studies: HPLC method development and validation. J. Pharm. Biomed. Anal. 2005, 39, 624-630. [CrossRef] 
21. Barakat, N.S. Evaluation of glycofurol-based gel as a new vehicle for topical application of Naproxen. AAPS PharmSciTech 2010, 11, 1138-1146. [CrossRef]

22. Fade, V. Link between drug absorption solubility and permeability measurements in Caco-2 cells. J. Pharm. Sci. 1998, 87, 1604-1607.

23. U.S. Environmental Protection Agency (EPA). (2007) Dermal Exposure Assessment: A Summary of EPA Approaches. Available online: https://cfpub.epa.gov/ncea/risk/recordisplay.cfm?deid=183584 (accessed on 13 May 2020).

24. OECD Environment, Health and Safety Publications, Guidance Notes on Dermal Absorption. Available online: https://www.oecd.org/chemicalsafety/testing/48532204.pdf (accessed on 13 May 2020).

25. Convention, T.U.S.P. Topical and Transdermal Drug Products-Product Performance Tests. Available online: http://dissolutiontech.com/DTresour/201011Articles/DT201011_A02.pdf (accessed on 18 May 2020).

26. OECD Guideline for the Testing of Chemicals 2 Draft Proposal for an Update of Test Guideline 4313 In Vitro Skin Corrosion: Reconstructed Human Epidermis (RhE) Test Method. Available online: https: //www.oecd.org/chemicalsafety/testing/43302385.pdf (accessed on 13 May 2020).

27. Health Effects Test Guidelines. Available online: https://www.epa.gov/test-guidelines-pesticides-and-toxicsubstances/series-870-health-effects-test-guidelines (accessed on 13 May 2020).

28. Todo, H. Transdermal permeation of drugs in various animal species. Pharmaceutics 2017, 9, 33. [CrossRef]

29. Karadzovska, D.; Riviere, J.E. Assessing vehicle effects on skin absorption using artificial membrane assays. Eur. J. Pharm. Sci. 2013, 50, 569-576. [CrossRef] [PubMed]

30. Uchida, T.; Kadhum, W.R.; Kanai, S.; Todo, H.; Oshizaka, T.; Sugibayashi, K. Prediction of skin permeation by chemical compounds using the artificial membrane, Strat-M ${ }^{\mathrm{TM}}$. Eur. J. Pharm. Sci. 2015, 67, 113-118. [CrossRef] [PubMed]

31. Montenegro, L.; Carbone, C.; Condorelli, G.; Drago, R.; Puglisi, G. Effect of oil phase lipophilicity on in vitro drug release from o/w microemulsions with low surfactant content. Drug Dev. Ind. Pharm. 2006, 32, 539-548. [CrossRef] [PubMed]

32. Mohammadi, T.; Saljoughi, E. Effect of production conditions on morphology and permeability of asymmetric cellulose acetate membranes. Desalination 2009, 243, 1-7. [CrossRef]

33. Kaur, L.; Singh, K.; Paul, S.; Singh, S.; Singh, S.; Jain, S.K. A Mechanistic Study to Determine the Structural Similarities Between Artificial Membrane Strat- $\mathrm{M}^{\mathrm{TM}}$ and Biological Membranes and Its Application to Carry Out Skin Permeation Study of Amphotericin B Nanoformulations. AAPS PharmSciTech 2018, 19, 1606-1624. [CrossRef]

34. Haq, A.; Dorrani, M.; Goodyear, B.; Joshi, V.; Michniak-Kohn, B. Membrane properties for permeability testing: Skin versus synthetic membranes. Int. J. Pharm. 2018, 539, 58-64. [CrossRef] 